PROCEEDINGS OF THE

AMERICAN MATHEMATICAL SOCIETY

Volume 137, Number 1, January 2009, Pages 11-18

S 0002-9939(08)09430-6

Article electronically published on July 18, 2008

\title{
UPPER BOUNDS FOR FINITE ADDITIVE 2-BASES
}

\author{
GANG YU
}

(Communicated by Ken Ono)

\begin{abstract}
For a positive integer $N$, a set $\mathcal{A} \subset[0, N] \cap \mathbb{Z}$ is called a 2-basis for $N$ if every integer $n \in[0, N]$ can be represented as $n=a+b$, where $a, b \in \mathcal{A}$. In this paper, we give a lower bound estimate for the cardinality of an additive 2-basis for $N$, as $N \rightarrow \infty$, which improves the existing results on this topic.
\end{abstract}

\section{INTRODUCTION}

For a positive integer $N$, an additive 2-basis (or simply a 2-basis) of size $k$ for $N$ is a set $\left\{0=a_{1}<a_{2}<\cdots<a_{k}\right\}$ of integers such that every positive integer up to $N$ can be represented as a sum of two elements of the set. A 2-basis $\mathcal{A}$ for $N$ is called restricted if $\mathcal{A} \subset[0, N / 2]$.

For a positive integer $N$, we denote by $k=k(N)$ (resp. $\left.k_{r}=k_{r}(N)\right)$ the smallest number of elements that can form a 2-basis (resp. a restricted 2-basis) for $N$. In this paper, we are interested in a lower bound estimation for $k$ and $k_{r}$, as $N \rightarrow \infty$. For this purpose, let

$$
\sigma:=\lim \sup _{N \rightarrow \infty} \frac{N}{(k(N))^{2}}, \quad \sigma_{r}:=\lim \sup _{N \rightarrow \infty} \frac{N}{\left(k_{r}(N)\right)^{2}} .
$$

Rohrbach 9] conjectured that $\sigma=\frac{1}{4}$, but this was disproved by many authors based on various constructions of thin 2-bases (cf. 2, 7]). In particular, Mrose 7 constructed a 2-basis $\mathcal{A} \subset[0, N]$ for every large $N$ with

$$
\frac{N}{|\mathcal{A}|^{2}} \geq \frac{2}{7}=0.285714 \ldots
$$

The exact value of $\sigma$ is still a mystery. Even a heuristic argument is still to be found in the literature that would suggest what the true value of $\sigma$ should be. Nevertheless, besides various constructions of thin 2-bases which yield lower bounds, there have been a number of results giving upper bounds for $\sigma$. For any 2-basis $\mathcal{A}$ for a positive integer $N$, a simple counting argument implies that

$$
N+1 \leq\left(\begin{array}{c}
|\mathcal{A}|+1 \\
2
\end{array}\right)
$$

which yields $\sigma \leq \frac{1}{2}$. This trivial bound for $\sigma$ has been improved as follows:

Received by the editors June 25, 2007, and, in revised form, November 15, 2007.

2000 Mathematics Subject Classification. Primary 11B13.

The author was supported by NSF grant DMS-0601033.

(C)2008 American Mathematical Society Reverts to public domain 28 years from publication 


$$
\begin{aligned}
& \sigma \leq 0.4992 \quad \text { (Rohrbach [9]) } \\
& \sigma \leq 0.4903 \quad \text { (Moser [5]) } \\
& \sigma \leq 0.4867 \quad \text { (Riddell [8]) } \\
& \sigma \leq 0.4847 \quad \text { (Moser, Pounder and Riddell [6]) } \\
& \sigma \leq 0.4802 \quad \text { (Klotz [4) } \\
& \sigma \leq 0.4789 \quad \text { (Güntürk and Nathanson [1]) }
\end{aligned}
$$

Among all these results, Rohrbach [9] attained his bound with a combinatorial argument, and all others used the Fourier series. In particular, Klotz [4] also appealed to Rohrbach's combinatorial method; Güntürk and Nathanson introduced the Fourier series for functions of two variables into the problem to obtain their improvement.

The restricted case has also been studied by several authors. Rohrbach 9 proved that $\sigma_{r} \leq 0.4654$. This was later improved by Riddell 8 ] to 0.43356 , and subsequently by Moser, Pounder and Riddell [6] to 0.42435 .

For a large integer $N$ and a 2-basis $\mathcal{A}$ for $N$, from the definition we know that $\mathcal{A}+\mathcal{A}$ covers all integers in the interval $[0, N]$. This determines asymptotically $2 N$ elements of $\mathcal{A}+\mathcal{A}$, yet gives a trivial estimate $|\mathcal{A}| \geq(\sqrt{2}+o(1)) \sqrt{N}$. Any better estimate for $|\mathcal{A}|$, no matter what method is used, essentially depends on the study of the irregular distribution of the elements of the sumset $\mathcal{A}+\mathcal{A}$ over the interval $[0,2 N]$ (or $[0, N]$ if $\mathcal{A}$ is restricted). Such irregularity is often captured by studying the values of the exponential sums over the set. Inspired by an idea involved in the author's recent work [10] on upper bounds for generalized Sidon sets, we shall study the distribution of the sumset $\mathcal{A}+\mathcal{A}$ over some subintervals of $[0,2 N]$ and derive from that a better upper bound for $\sigma$.

Theorem 1.1. We have

$$
\sigma \leq 0.46972 .
$$

With a similar method, we also get an improvement for $\sigma_{r}$.

Theorem 1.2. We have

$$
\sigma_{r} \leq \frac{7+\sqrt{5}}{22}=0.41982 \ldots
$$

Notation. Throughout this paper, $A \gtrsim B$ (resp. $A \lesssim B$ ) means that $A \geq(1+o(1)) B$ (resp. $A \leq(1+o(1)) B)$ as $N \rightarrow \infty$.

\section{Preliminaries}

For a finite set $\mathcal{B} \subset \mathbb{Z}$, we denote by $f_{\mathcal{B}}(\beta)$ the generating function of $\mathcal{B}$ as

$$
f_{\mathcal{B}}(\beta)=\sum_{b \in \mathcal{B}} e(\beta b), \quad \text { where } e(t)=\exp (2 \pi i t) .
$$

We also define, for any $n \in \mathbb{Z}$, that

$$
r_{\mathcal{B}}(n):=\#\{(a, b) \in \mathcal{B} \times \mathcal{B}: a+b=n\}
$$

and

$$
d_{\mathcal{B}}(n):=\#\{(a, b) \in \mathcal{B} \times \mathcal{B}: a-b=n\} .
$$


The following lemma (Lemma 2.11) is fundamental in our proofs of Theorems 1.1 and 1.2. It is a more general form of Lemma 2 in [10. We believe that such a result is of independent interest and will be useful in other places. Thus we give the lemma in a more general setting even though the proofs of the theorems will only need it with a very special weight function.

Let $u(x)$ be a non-negative function supported on $[0,1]$, with piecewise continuous derivative and bounded total variation, and

$$
\int_{0}^{1} u(t) d t=1 \text {. }
$$

Let $W(x)$ be the even function on $[-1,1]$ defined by

$$
W(x):=\int_{0}^{1-|x|} u(t) u(t+|x|) d t .
$$

For given real numbers $p, \delta$ with $p>2 \delta>0$, let $w_{p, \delta}(x)$ be the periodic function on $(-\infty,+\infty)$ which, on a period $[-p / 2, p / 2]$, is defined by

$$
w_{p, \delta}(x)=\left\{\begin{array}{cl}
W(x / \delta) & \text { if }|x| \leq \delta, \\
0 & \text { if } \delta<|x| \leq \frac{1}{2} p .
\end{array}\right.
$$

Lemma 2.1. Suppose $\mathcal{B} \subset[0, N] \cap \mathbb{Z}$, and $w_{p, \delta}(x)$ is given by (2.1). Let

$$
D_{p, \delta}(\mathcal{B})=\sum_{m=-N}^{N} w_{p, \delta}(m / N) d_{\mathcal{B}}(m)
$$

and

$$
R_{p, \delta, \kappa}(\mathcal{B})=\sum_{m=0}^{2 N} w_{p, \delta}(m / N+\kappa) r_{\mathcal{B}}(m) .
$$

Then for any fixed real number $\kappa$, we have

$$
D_{p, \delta}(\mathcal{B})+R_{p, \delta, \kappa}(\mathcal{B}) \geq \frac{2 \delta}{p}|\mathcal{B}|^{2}
$$

and

$$
D_{p, \delta}(\mathcal{B}) \geq R_{p, \delta, \kappa}(\mathcal{B}) .
$$

Proof. We first note that $w_{p, \delta}(x)$ has a formal Fourier expansion into the cosine series

$$
w_{p, \delta}(x)=\frac{a_{p, \delta}(0)}{2}+\sum_{n=1}^{\infty} a_{p, \delta}(n) \cos (2 n \pi x / p),
$$

where, for the integer $n \geq 0$,

$$
a_{p, \delta}(n)=\frac{2}{p} \int_{-\frac{p}{2}}^{\frac{p}{2}} w_{p, \delta}(x) \cos (2 n \pi x / p) d x .
$$

By a straightforward calculation, we get

$$
a_{p, \delta}(n)=\frac{2 \delta}{p}\left\{\left(\int_{0}^{1} u(t) \cos (2 n \pi \delta t / p) d t\right)^{2}+\left(\int_{0}^{1} u(t) \sin (2 n \pi \delta t / p) d t\right)^{2}\right\}
$$

from which we see that

$$
\frac{a_{p, \delta}(0)}{2}=\frac{\delta}{p}, \quad a_{p, \delta}(n) \geq 0 \quad \text { for } \quad n \geq 1
$$


and that the Fourier series (2.4) converges uniformly to $w_{p, \delta}(x)$. From (2.4) and (2.5), we thus have

$$
\begin{aligned}
D_{p, \delta}(\mathcal{B}) & =\sum_{m=-N}^{N} d_{\mathcal{B}}(m)\left(\frac{\delta}{p}+\sum_{n=1}^{\infty} a_{p, \delta}(n) \cos \left(\frac{2 n m \pi}{p N}\right)\right) \\
& =\frac{\delta}{p} \sum_{m=-N}^{N} d_{\mathcal{B}}(m)+\sum_{n=1}^{\infty} a_{p, \delta}(n) \sum_{m=-N}^{N} d_{\mathcal{B}}(m) \cos \left(\frac{2 n m \pi}{p N}\right) \\
& =\frac{\delta}{p}|\mathcal{B}|^{2}+\sum_{n=1}^{\infty} a_{p, \delta}(n)\left|f_{\mathcal{B}}\left(\frac{n}{p N}\right)\right|^{2}
\end{aligned}
$$

and

$$
\begin{aligned}
R_{p, \delta, \kappa}(\mathcal{B}) & =\sum_{m=0}^{2 N} r_{\mathcal{B}}(m)\left(\frac{\delta}{p}+\sum_{n=1}^{\infty} a_{p, \delta}(n) \cos \left(\frac{2 n(m+\kappa N) \pi}{p N}\right)\right) \\
& =\frac{\delta}{p} \sum_{m=0}^{2 N} r_{\mathcal{B}}(m)+\sum_{n=1}^{\infty} a_{p, \delta}(n) \sum_{m=0}^{2 N} r_{\mathcal{B}}(m) \cos \left(\frac{2 n(m+\kappa N) \pi}{p N}\right) \\
& =\frac{\delta}{p}|\mathcal{B}|^{2}+\sum_{n=1}^{\infty} a_{p, \delta}(n) \Re\left(f_{\mathcal{B}}{ }^{2}\left(\frac{n}{p N}\right) e\left(\frac{\kappa n}{p}\right)\right) .
\end{aligned}
$$

From (2.6), (2.7), we thus have

$$
D_{p, \delta}(\mathcal{B})+R_{p, \delta, \kappa}(\mathcal{B})=\frac{2 \delta}{p}|\mathcal{B}|^{2}+\sum_{n=1}^{\infty} a_{p, \delta}(n)\left(\left|f_{\mathcal{B}}\left(\frac{n}{p N}\right)\right|^{2}+\Re\left(f_{\mathcal{B}}{ }^{2}\left(\frac{n}{p N}\right) e\left(\frac{\kappa n}{p}\right)\right)\right) .
$$

Note that, for each $n \geq 1$, we have $a_{p, \delta}(n) \geq 0$ and

$$
\left|f_{\mathcal{B}}\left(\frac{n}{p N}\right)\right|^{2}+\Re\left(f_{\mathcal{B}}^{2}\left(\frac{n}{p N}\right) e\left(\frac{\kappa n}{p}\right)\right) \geq 0 ;
$$

(2.2) then follows. It is also clear that (2.3) holds for any $\kappa$ from (2.6), (2.7), and the fact that $a_{p, \delta}(n) \geq 0$ for all positive integers $n$.

Lemma 2.2. Suppose $\mathcal{A}$ is a 2-basis for a sufficiently large integer $N$. Then for any integer $n$ not divisible by $N$, we have

$$
\left|f_{\mathcal{A}}(n / N)\right| \lesssim \sqrt{|\mathcal{A}|^{2}-2 N}
$$

Proof. When $N \nmid n$, we have

$$
\sum_{m=0}^{N-1} e(n m / N)=0
$$

From this we get

$$
f_{\mathcal{A}}^{2}(n / N)=\sum_{m=0}^{2 N} r_{\mathcal{A}}(m) e(m n / N)=\sum_{m=0}^{2 N} r_{\mathcal{A}}^{*}(m) e(m n / N),
$$

where

$$
r_{\mathcal{A}}^{*}(m)=\left\{\begin{array}{cl}
r_{\mathcal{A}}(m)-2 & \text { if } 0 \leq m \leq N-1, \\
r_{\mathcal{A}}(m) & \text { otherwise }
\end{array}\right.
$$


Since $\mathcal{A}$ is a 2-basis for $N$, we have $r_{\mathcal{A}}{ }^{*}(m) \geq 0$ for all but at most $O(|\mathcal{A}|)$ integers $m \in[0, N-1]$. Thus

$$
\left|f_{\mathcal{A}}^{2}(n / N)\right|^{2} \leq \sum_{m=0}^{2 N} r_{\mathcal{A}}^{*}(m)+O(|\mathcal{A}|) \leq|\mathcal{A}|^{2}-2 N+O(|\mathcal{A}|) .
$$

The lemma then follows from the fact that $|\mathcal{A}|^{2}>(2+\varepsilon) N$ for some $\epsilon>0$ as $N \rightarrow \infty$.

In our application of Lemma 2.1 to the proofs of Theorems 1.1 and 1.2, we take $u(t)=1$ on $[0,1]$. Thus

$$
w_{p, \delta}(x)=\left\{\begin{array}{cl}
1-|x| / \delta & \text { if }|x| \leq \delta \\
0 & \text { if } \delta<|x| \leq \frac{1}{2} p .
\end{array}\right.
$$

In the next lemma and henceforth, we shall suppose $w_{p, \delta}(x)$ given by (2.10). We note that in the cosine series (2.4) we then have the following, which is more precise than (2.5):

$$
a_{p, \delta}(n)=\frac{p}{\delta(n \pi)^{2}}(1-\cos (2 n \pi \delta / p)) \geq 0 \quad \text { for } n \geq 1, \quad \text { and } \quad \sum_{n=1}^{\infty} a_{p, \delta}(n)=1-\frac{\delta}{p} .
$$

Lemma 2.3. Suppose $0<\delta \leq \frac{1}{2}$. Suppose $\mathcal{A}$ is a 2-basis for a sufficiently large integer $N$, and $D_{p, \delta}(\mathcal{A})$ is as defined in Lemma 2.1, with $w_{p, \delta}(x)$ given by (2.10). If $p \geq 1$, then we have

$$
D_{p, \delta}(\mathcal{A}) \leq D_{1, \delta}(\mathcal{A}) \lesssim|\mathcal{A}|^{2}-2(1-\delta) N .
$$

Furthermore, if $\mathcal{A}$ is a restricted 2-basis for $N$, and $p \geq \frac{1}{2}+\delta$, then (2.12) holds as well.

Proof. Note that if $p \geq 1$, then

$$
w_{p, \delta}(x) \leq w_{1, \delta}(x) \text { for } \quad x \in[-1,1],
$$

which yields

$$
D_{p, \delta}(\mathcal{A}) \leq D_{1, \delta}(\mathcal{A}) \quad \text { if } \quad p \geq 1 .
$$

Now from (2.6), (2.11), and Lemma 2.2, we have

$$
\begin{aligned}
D_{1, \delta}(\mathcal{A}) & =\delta|\mathcal{A}|^{2}+\sum_{\substack{n=1 \\
N \nmid n}}^{\infty} a_{1, \delta}(n)\left|f_{\mathcal{A}}\left(\frac{n}{N}\right)\right|^{2}+\sum_{n=1}^{\infty} a_{1, \delta}(n N)|\mathcal{A}|^{2} \\
& \lesssim \delta|\mathcal{A}|^{2}+(1-\delta)\left(|\mathcal{A}|^{2}-2 N\right)+O\left(\frac{|\mathcal{A}|^{2}}{N^{2} \delta}\right) \\
& \lesssim \delta \delta|\mathcal{A}|^{2}-2(1-\delta) N
\end{aligned}
$$

which, along with (2.13), proves (2.12).

If $\mathcal{A}$ is a restricted 2-basis for $N$, and $p \geq \frac{1}{2}+\delta$, then we have $D_{p, \delta}(\mathcal{A})=D_{1, \delta}(\mathcal{A})$ since in both sums $m \in[-N / 2, N / 2]$ and $w_{p, \delta}(x)=w_{1, \delta}(x)$ for $x \in[-1 / 2,1 / 2]$. 


\section{Proof of Theorem 1.1}

In this section, we shall use the lemmas to prove Theorem 1.1 Suppose $\mathcal{A}$ is a 2-basis for a sufficiently large integer $N$. Without loss of generality, we shall assume $|\mathcal{A}| \asymp \sqrt{N}$. Let $\delta, \varepsilon$ be real numbers satisfying $0<\varepsilon<\delta<\frac{1}{2}$, the actual values to be determined later, and $w_{p, \delta}(x)$ be given by (2.10). From Lemma 2.1 we have

$$
D_{1+2(\delta-\varepsilon), \delta}(\mathcal{A})+R_{1+2(\delta-\varepsilon), \delta, \delta-\varepsilon}(\mathcal{A}) \geq \frac{2 \delta}{1+2(\delta-\varepsilon)}|\mathcal{A}|^{2}
$$

and

$$
D_{1+3 \delta-\varepsilon, \delta}(\mathcal{A})+R_{1+3 \delta-\varepsilon, \delta, \delta}(\mathcal{A}) \geq \frac{2 \delta}{1+3 \delta-\varepsilon}|\mathcal{A}|^{2} .
$$

Combining these with Lemma 2.3 we see that $R_{1+2(\delta-\varepsilon), \delta, \delta-\varepsilon}(\mathcal{A})+R_{1+3 \delta-\varepsilon, \delta, \delta}(\mathcal{A})$ is

$$
\begin{aligned}
& \gtrsim \frac{2 \delta}{1+2(\delta-\varepsilon)}|\mathcal{A}|^{2}+\frac{2 \delta}{1+3 \delta-\varepsilon}|\mathcal{A}|^{2}-2 D_{1, \delta}(\mathcal{A}) \\
& \gtrsim\left(\frac{2 \delta}{1+2(\delta-\varepsilon)}+\frac{2 \delta}{1+3 \delta-\varepsilon}-2\right)|\mathcal{A}|^{2}+4(1-\delta) N .
\end{aligned}
$$

Now we notice that

$$
R_{1+2(\delta-\varepsilon), \delta, \delta-\varepsilon}(\mathcal{A})=R_{1+2(\delta-\varepsilon), \delta, \delta-\varepsilon}^{(1)}(\mathcal{A})+R_{1+2(\delta-\varepsilon), \delta, \delta-\varepsilon}^{(2)}(\mathcal{A}),
$$

where

$$
R_{1+2(\delta-\varepsilon), \delta, \delta-\varepsilon}^{(1)}(\mathcal{A})=\sum_{m \leq \varepsilon N} \frac{\varepsilon}{\delta}\left(1-\frac{m}{\varepsilon N}\right) r_{\mathcal{A}}(m)+\sum_{(1-\varepsilon) N<m \leq N} \frac{\varepsilon}{\delta}\left(1-\frac{N-m}{\varepsilon N}\right) r_{\mathcal{A}}(m)
$$

and

$$
R_{1+2(\delta-\varepsilon), \delta, \delta-\varepsilon}^{(2)}(\mathcal{A})=\sum_{N<m \leq(1+2 \delta-\varepsilon) N}\left(1-\frac{|m-(1+\delta-\varepsilon) N|}{\delta N}\right) r_{\mathcal{A}}(m) .
$$

From these and the fact that

$$
R_{1+3 \delta-\varepsilon, \delta, \delta}(\mathcal{A})=\sum_{(1+\delta-\varepsilon) N<m \leq(1+3 \delta-\varepsilon) N}\left(1-\frac{|m-(1+2 \delta-\varepsilon) N|}{N}\right) r_{\mathcal{A}}(m),
$$

we have

$$
R_{1+2(\delta-\varepsilon), \delta, \delta-\varepsilon}(\mathcal{A})+R_{1+3 \delta-\varepsilon, \delta, \delta}(\mathcal{A}) \leq R_{1+2(\delta-\varepsilon), \delta, \delta-\varepsilon}^{(1)}(\mathcal{A})+\sum_{N<m \leq 2 N} r_{\mathcal{A}}(m) .
$$

Since $\mathcal{A}$ is a 2-basis for $N$ and since the coefficient of $r_{\mathcal{A}}(m)$ for each $m$ in $R_{1+2(\delta-\varepsilon), \delta, \delta-\varepsilon}^{(1)}(\mathcal{A})$ is less than 1 , we see that, apart from an error term of at most $O(|\mathcal{A}|)$,

$$
\begin{aligned}
& \sum_{m=0}^{N} r_{\mathcal{A}}(m)-R_{1+2(\delta-\varepsilon), \delta, \delta-\varepsilon}^{(1)}(\mathcal{A}) \\
& \quad \gtrsim 2 N-2\left(\sum_{m \leq \varepsilon N} \frac{\varepsilon}{\delta}\left(1-\frac{m}{\varepsilon N}\right)+\sum_{(1-\varepsilon) N<m \leq N} \frac{\varepsilon}{\delta}\left(1-\frac{N-m}{\varepsilon N}\right)\right) \\
& \quad \gtrsim\left(2-\frac{2 \varepsilon^{2}}{\delta}\right) N .
\end{aligned}
$$


Now, from (3.1), (3.2), and (3.3), we get

$$
\begin{aligned}
|\mathcal{A}|^{2} & =\sum_{m=0}^{N} r_{\mathcal{A}}(m)+\sum_{m=N+1}^{2 N} r_{\mathcal{A}}(m) \\
& \gtrsim R_{1+2(\delta-\varepsilon), \delta, \delta-\varepsilon}^{(1)}(\mathcal{A})+\left(2-\frac{2 \varepsilon^{2}}{\delta}\right) N+\sum_{N<m \leq 2 N} r_{\mathcal{A}}(m) \\
& \gtrsim\left(\frac{2 \delta}{1+2(\delta-\varepsilon)}+\frac{2 \delta}{1+3 \delta-\varepsilon}-2\right)|\mathcal{A}|^{2}+4(1-\delta) N+\left(2-\frac{2 \varepsilon^{2}}{\delta}\right) N,
\end{aligned}
$$

which gives

$$
\frac{|\mathcal{A}|^{2}}{N} \gtrsim \frac{6-4 \delta-\frac{2 \varepsilon^{2}}{\delta}}{3-\frac{2 \delta}{1+2(\delta-\varepsilon)}-\frac{2 \delta}{1+3 \delta-\varepsilon}} .
$$

Let $\delta=0.2257$ and $\varepsilon=0.0882$ in (3.4), then we have

$$
\frac{|\mathcal{A}|^{2}}{N} \gtrsim 2.12893875>\frac{1}{0.46972},
$$

which proves Theorem 1.1

\section{Proof of Theorem 1.2}

Suppose $\mathcal{A}$ is a restricted 2-basis for a sufficiently large integer $N$. (Thus $\mathcal{A} \subset$ $[0, N / 2]$.) Let $\delta \in[1 / 6,1 / 2]$. From (2.3) and Lemma 2.3, we have

$$
R_{\frac{1}{2}+\delta, \delta, \frac{1}{2} \delta-\frac{1}{4}}(\mathcal{A}) \leq D_{\frac{1}{2}+\delta, \delta}(\mathcal{A}) \lesssim|\mathcal{A}|^{2}-2(1-\delta) N .
$$

On the other hand, since $\frac{1}{6} \leq \delta \leq \frac{1}{2}$, we see that

$$
\begin{aligned}
R_{\frac{1}{2}+\delta, \delta, \frac{1}{2} \delta-\frac{1}{4}}(\mathcal{A}) & \sum_{0 \leq m \leq\left(\frac{1}{4}+\frac{\delta}{2}\right) N}\left(1-\frac{\left|m-\left(\frac{1}{4}-\frac{\delta}{2}\right) N\right|}{\delta N}\right) r_{\mathcal{A}}(m) \\
& +\sum_{\left(\frac{3}{4}-\frac{\delta}{2}\right) N \leq m \leq N}\left(1-\frac{\left|m-\left(\frac{3}{4}+\frac{\delta}{2}\right) N\right|}{\delta N}\right) r_{\mathcal{A}}(m) \\
\gtrsim & \sum_{0 \leq m \leq\left(\frac{1}{4}+\frac{\delta}{2}\right) N}\left(1-\frac{\left|m-\left(\frac{1}{4}-\frac{\delta}{2}\right) N\right|}{\delta N}\right) \\
& +2 \sum_{\left(\frac{3}{4}-\frac{\delta}{2}\right) N \leq m \leq N}\left(1-\frac{\left|m-\left(\frac{3}{4}+\frac{\delta}{2}\right) N\right|}{\delta N}\right) \\
\gtrsim & \left(4 \delta-\frac{2\left(\frac{3}{2} \delta-\frac{1}{4}\right)^{2}}{\delta}\right) N .
\end{aligned}
$$

Thus from this and (4.1), we get

$$
\frac{|\mathcal{A}|^{2}}{N} \gtrsim 2+2 \delta-\frac{2\left(\frac{3}{2} \delta-\frac{1}{4}\right)^{2}}{\delta}:=g(\delta) .
$$

Theorem 1.2 then follows by noticing that $\frac{\sqrt{5}}{10} \in\left[\frac{1}{6}, \frac{1}{2}\right]$ and $\frac{1}{g(\sqrt{5} / 10)}=\frac{7+\sqrt{5}}{22}$. 


\section{A FURTHER REMARK}

When estimating the size of a set $\mathcal{A} \subset[0, N] \cap \mathbb{Z}$ in an order 2 additive problem, such as a 2-basis for $N$ or a $B_{2}[g]$ set (a type of generalized Sidon set), it seems more natural to use the Fourier series of functions of two variables rather than a single variable function, at least in some special cases. For instance, to give an explicit upper bound for a weighted sum like

$$
\sum_{m \in \mathcal{I}(N)} w(m / N) r_{\mathcal{A}}(m)=\sum_{\substack{a, b \in \mathcal{A} \\ a+b \in \mathcal{I}(N)}} w\left(\frac{a+b}{N}\right)
$$

with $\mathcal{I}(N)$ being a short interval close to 0 or $2 N$, we can express the sum as a trigonometric series in accordance with the Fourier expansion of the function $f(x, y)=w(x+y)$ on $[0,1] \times[0,1]$ or that of $w(x)$ on $[0,2]$. Comparing the sum with the Fourier expansion of $w(x)$, the trigonometric sum resulting from the Fourier series of the 2 variable function $f(x, y)$ has a smaller "constant term", and its cosine (and/or sine) terms have period 1 and are thus related to $f_{\mathcal{A}}\left(\frac{n}{N}\right)$ (rather than $f_{\mathcal{A}}\left(\frac{n}{2 N}\right)$ ). Such sums (with $N \nmid n$ ) have presumably smaller sizes than $f_{\mathcal{A}}\left(\frac{m}{2 N}\right)(2 \nmid m)$ have in the 2-basis problem (and a smaller size on average in the $B_{2}[g]$ problem). This means that, if one could find a weight function $w$ such that the Fourier series of $w(x+y)$ has a small $L^{1}$-norm, then presumably a better lower bound should be obtained for 2-bases of integers; and a better upper bound for $B_{2}[g]$-sets would be achieved if the $L^{\frac{4}{3}}$-norm of the Fourier series is small. The difficulty of this approach is to find a (more or less) optimal weight function satisfying the properties we want. This surely requires a better understanding of the Fourier coefficients of 2 -variable functions.

\section{ACKNOWLEDGEMENT}

The author is grateful to Richard Aron for his helpful comments and suggestions.

\section{REFERENCES}

[1] C. Güntürk and M. Nathanson, A new upper bound for finite additive bases, Acta Arith., 124(2006), 235-255. MR2250418(2007f:11012)

[2] N. Hämmerer and G. Hofmeister, Zu einer Vermutung von Rohrbach, J. Reine Angew. Math., 286/287(1976), 239-247. MR0422189 (54:10181)

[3] G. Hofmeister, Thin bases of order two, J. Number Theory, 86(2001), 118-132. MR.1813532 (2001m:11018)

[4] W. Klotz, Eine obere Schranke für die Reichweite einer Extremalbasis zweiter Ordnung, J. Reine Angew. Math., 238(1969), 161-168. MR0246848(40:117)

[5] L. Moser, On the representation of $1,2, \ldots, n$ by sums, Acta Arith., 6(1960), 11-13. MR.0122800 (23:A133)

[6] L. Moser, J. Pounder and J. Riddell, On the cardinality of h-bases for $n$, J. London Math. Soc., 44(1969), 397-407. MR0238798 (39:162)

[7] A. Mrose, Untere Schranken für die Reichweiten von Extremalbasen fester Ordnung, Abh. Math. Sem. Univ. Hamburg, 48(1979), 118-124. MR537452 (80g:10058)

[8] J. Riddell, On bases for sets of integers, Master's Thesis, University of Alberta, 1960.

[9] H. Rohrbach, Ein Beitrag zur additiven Zahlentheorie, Math. Z., 42(1937), 1-30. MR 1545658

[10] G. Yu, An upper bound for $B_{2}[g]$ sets, J. Number Theory, 122(2007), no. 1, 211-220. MR2287120(2008a:11012)

Department of Mathematical Sciences, Kent State University, Kent, Ohio 44242

E-mail address: yu@math.kent.edu 